\title{
ПРАКТИЧЕСКАЯ СХЕМА
}

\section{С РАСШИРЕННОЙ ПОЛОСОЙ ЧАСТОТ НА ОСНОВЕ СВЧ МИС УСИЛИТЕЛЕЙ} МОЩНОСТИ

\author{
Э.Колон, Р.Сетти, М.Мордкович, В.Ченг, Дж.Куинн, Дж.Ньюболд, Б.Каплан
}

УДК 621.375 .026 BAK 05.27.00

\begin{abstract}
Акцент рынка на диапазонах частот, используемых в беспроводных коммуникациях, привел к тому, что полупроводниковая промышленность сосредоточилась на этих узких диапазонах. В результате стало ослабевать внимание к характеристикам усилителей мощности на базе СВЧ монолитных интегральных схем (МИС) в широких диапазонах частот. Однако узкополосные схемы плохо применимы для таких широкополосных приложений, как радиосвязь в ОВЧ- и УВЧ-диапазонах, контрольно-измерительная аппаратура, программно-определяемое радио и др. В данной статье рассматривается практическая схема с применением усилителя мощности GVA-91+ компании Mini-Circuits и приводятся результаты измерений, показывающие отличные характеристики усилителя в широком диапазоне частот.
\end{abstract}

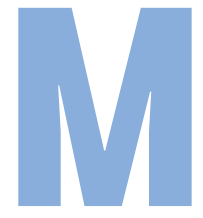

одель GVA-91+ представляет собой усилитель мощности с высоким КПД на основе арсенид-галлиевых биполярных гетеротранзисторов (НВТ), выполненный в стандартном корпусе SOT-89. GVA-91+ обладает отличными характеристиками в диапазонах сотовой связи, в том числе LTE. С помощью согласующих цепей этот усилитель может обеспечить выходную мощность при компрессии 1 дБ (PldB), равную 29 дБм на частотах от 869 до 960 МГц и 27 дБм в диапазоне от 2110 до 2170 МГц. Такая высокая выходная мощность сочетается с КПД по добавленной мощности (РАЕ) от 41 до 54\% в диапазонах частот, для которых обеспечено согласование. Однако данный усилитель разрабатывался таким образом, чтобы достигались высокие характеристики в узких частотных диапазонах, определяемых согласующими схемами.

Для того чтобы расширить область применения усилителя, компания Mini-Circuits разработала новую практическую схему, в которой усилители GVA-91+ применяются в дифференциальной конфигурации (рис.1, 2). Использование квадратурных гибридных ответвителей обеспечивает отличное согласование, ограниченное только характеристиками согласования данных ответвителей. Теоретически, любая отраженная волна, вызванная рассогласованием, вносимым усилителями (как на входе, так и на выходе), будет рассеиваться в нагрузке изолированного порта квадратурных гибридных ответвителей. Кроме того, дифференциальная конфигурация должна обеспечить увеличение выходной мощности на 3 дБ по сравнению с отдельными усилителями при сохранении того же коэффициента усиления".

В предложенной схеме усилитель GVA-91+ используется за пределами того частотного диапазона, для

\footnotetext{
Maas, Stephen A. Nonlinear Microwave and RF Circuits. 2nd ed. Boston, MA: Artech House, 2003.
} 


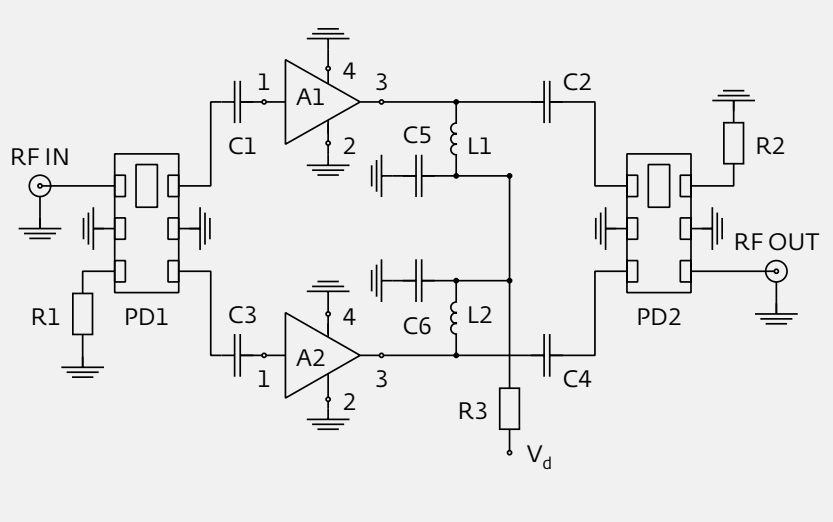

Рис.1. Дифференциальная схема усилителя на основе GVA-91+

которого имелись схемы согласования, что является примером возможности практического применения данного подхода. Для того чтобы обеспечить небольшой размер устройства, были использованы квадратурные гибридные ответвители серии QCN компании Mini-Circuits, выполненные в корпусе 1206 по технологии низкотемпературной совместно обжигаемой керамики (LTCC). Компания Mini-Circuits в настоящее время предлагает 34 серийно выпускаемых модели серии QCN, охватывающие диапазон частот от 330 до 4500 МГц, при этом каждая модель перекрывает диапазон, примерно соответствующий октаве. Полоса пропускания рассматриваемой схемы на базе GVA-91+ определяется в основном полосой

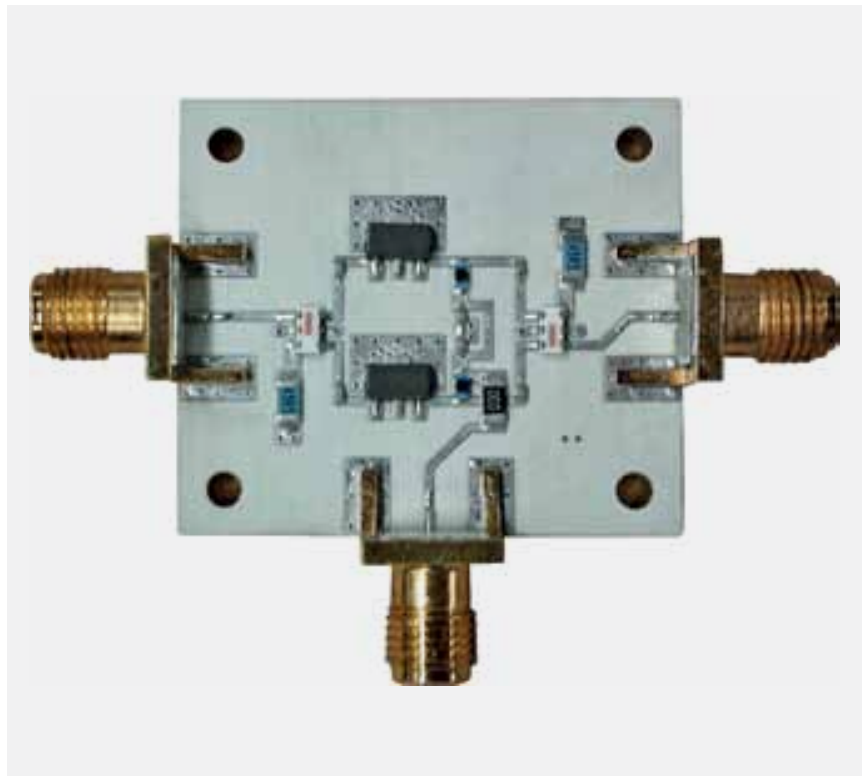

Рис.2. Прототип практической дифференциальной схемы усилителя на основе GVA-91+
Характеристики элементов схемы, представленной на рис.1

\begin{tabular}{|l|l|l|}
\hline Компонент & Номинал / тип & $\begin{array}{l}\text { Размер / } \\
\text { корпус }\end{array}$ \\
\hline A1, A2 & Mini-Circuits GVA-91+ & SOT-89 \\
\hline PD1, PD2 & Mini-Circuits QCN-5+ & 1206 \\
\hline L1, L2 & 180 нГн & 0603 \\
\hline C1-C6 & 0,001 мкФ & 0402 \\
\hline R1, R2 & 49,9 Ом & 1206 \\
\hline R3 & 0 Ом & 1206 \\
\hline
\end{tabular}

пропускания гибридных ответвителей, что позволяет применять усилитель в различных частотных диапазонах.

Прототипы схемы были собраны на печатных платах с использованием квадратурных гибридных ответвителей QCN-5+. Такая конфигурация обеспечивает работу в диапазоне 330-580 МГц, соответствующем номинальной полосе пропускания QCN-5+. Прототипы были изготовлены в виде микрополосковых схем с импедансом 50 Ом на основании из материала Rogers 4350В толщиной 0,010".

Малосигнальные S-параметры измерялись при свипировании частоты в диапазоне от 300 до 600 МГц (рис.3, 4, 5). Опорные плоскости измерений были

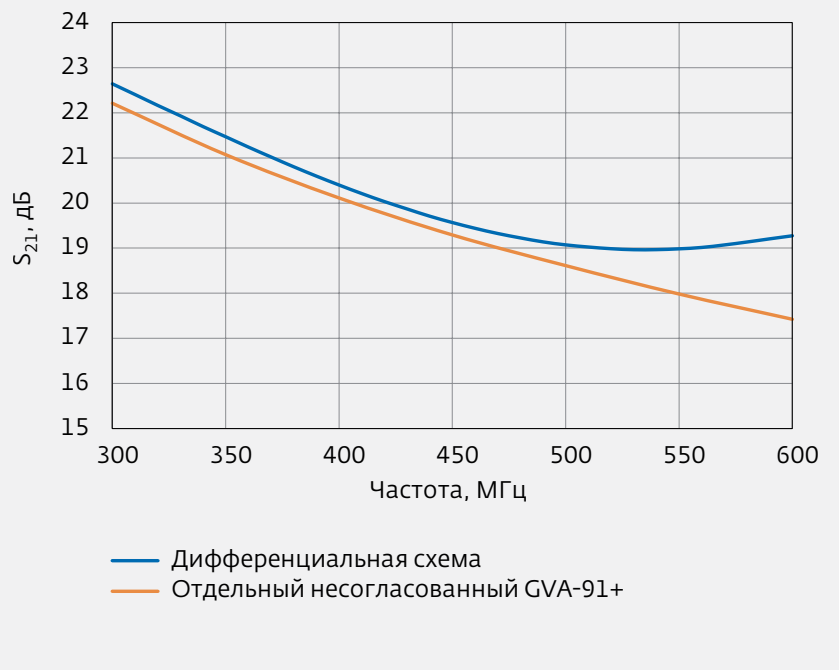

Рис.3. Сравнение коэффициентов усиления дифференциальной схемы и отдельного несогласованного усилителя GVA-91+ при малом сигнале 


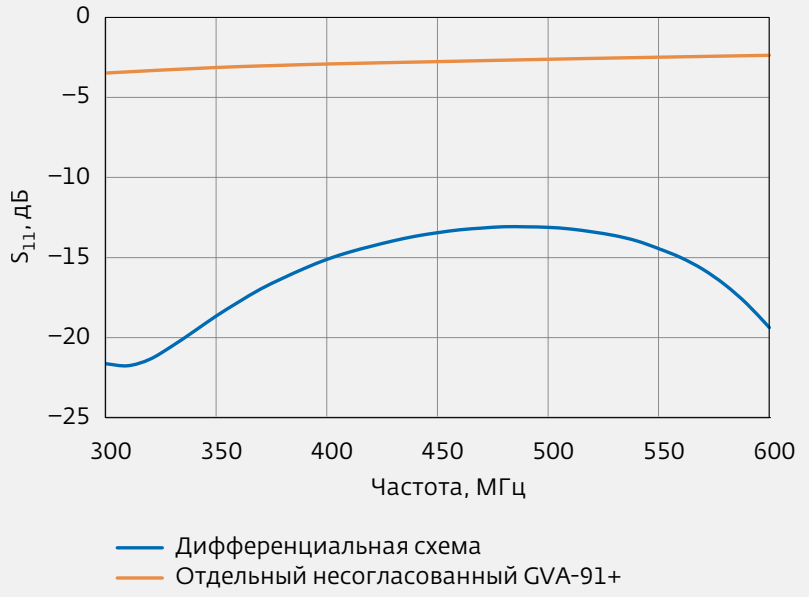

Рис.4. Сравнение обратных потерь на входе дифференциальной схемы и отдельного несогласованного усилителя GVA-91+

выбраны в месте стыковки соединителей SMA и коаксиальных кабелей, которыми тестируемое устройство подсоединялось к векторному анализатору цепей, использовавшемуся в экспериментах. На графиках приведено сравнение результатов при малом сигнале, полученных для рассматриваемой в статье схемы, с данными для отдельного усилителя GVA-91+ без внешней согласующей цепи. Из рис.3 видно, что

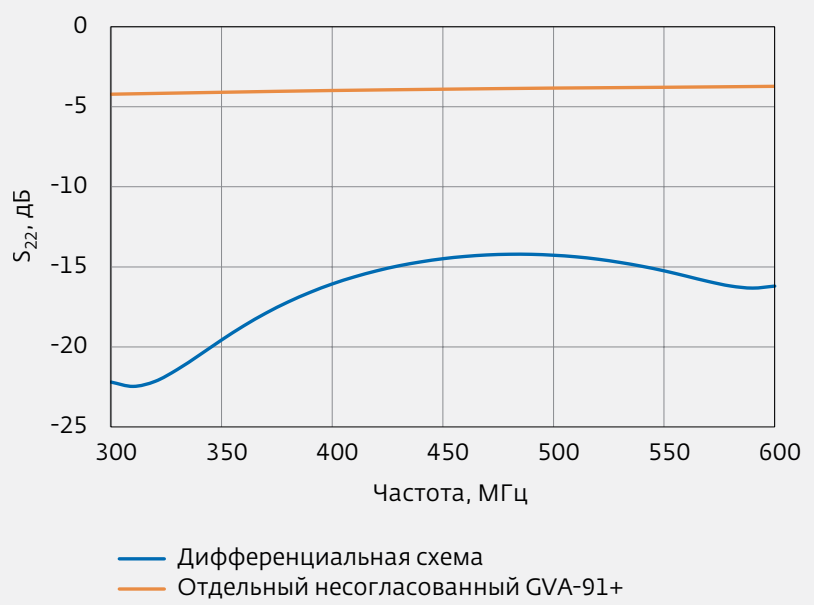

Рис.5. Сравнение обратных потерь на выходе дифференциальной схемы и отдельного несогласованного усилителя GVA-91+

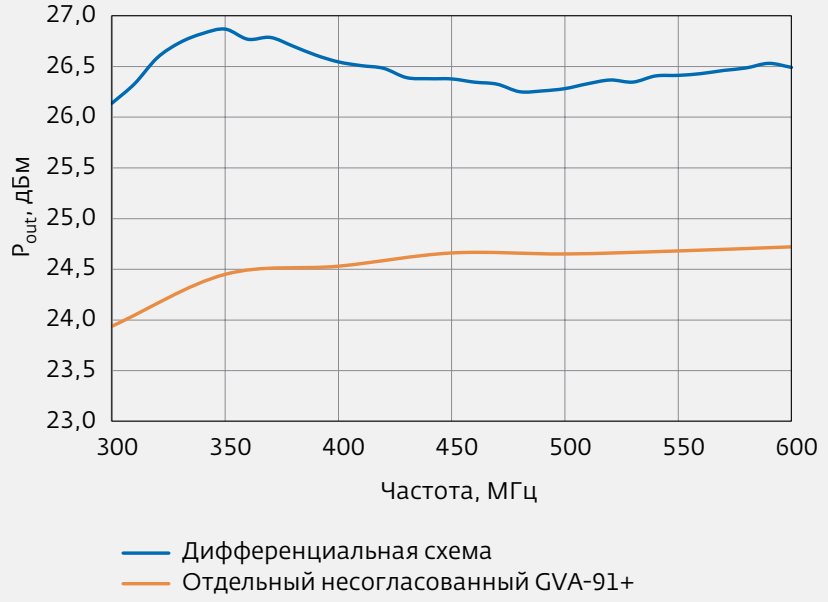

Рис.6. Сравнение выходной мощности при компрессии 1 дБ дифференциальной схемы и отдельного несогласованного усилителя GVA-91+

рассогласование в случае отдельных усилителей приводит к ухудшению их амплитудной характеристики. Усиление малого сигнала при дифференциальной конфигурации немного выше, чем при применении отдельного усилителя. Как видно из рис.4 и 5, обратные потери на входе и выходе при дифференциальной конфигурации значительно меньше: в диапазоне октавы они находятся на уровне -13 дБ и ниже. Еще

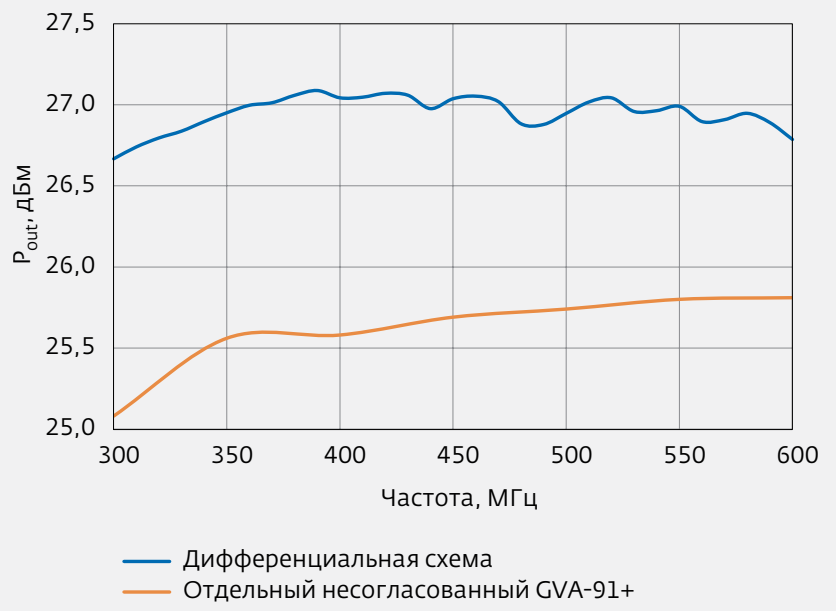

Рис.7. Сравнение выходной мощности при компрессии 3 дБ дифференциальной схемы и отдельного несогласованного усилителя GVA-91+ 
одно преимущество предложенной схемы - повышение выходной мощности усилителя. На рис.6 и 7 показано, что при компрессии 1 и 3 дБ в диапазоне от 300 до 600 МГц данный параметр примерно на 2 дБ выше, чем у отдельного усилителя GVA-91+.

Ограничения частотного диапазона рассматриваемой дифференциальной схемы обусловлены асимметрией амплитуды и фазы, присущей неидеальным квадратурным гибридным ответвителям. Другой негативный побочный эффект данной схемы - низкое значение ее результирующего КПД по добавленной мощности. Он составляет $27 \%$ на частоте 500 МГц против 45\% на частоте 920 МГц у отдельного GVA-91+. Однако это значение все же выше, чем то, которое можно достичь с помощью других конфигураций широкополосного усилителя, таких как схема Дарлингтона.

Таким образом, предложенное решение открывает для разработчиков возможности более гибкого применения усилителя GVA-91+ и других СBЧ МИС усилителей в корпусе SOT-89, обычно требующих узкополосного согласования, но также способных обеспечить высокие характеристики в более широких диапазонах частот. Кроме того, такие же схемы можно применять для увеличения выходной мощности устройств на основе усилителей в корпусе SOT-89, предназначенных специально для работы в широких частотных диапазонах, таких как компоненты серий РНА, PGA и GALI компании Mini-Circuits. Различные квадратурные гибридные ответвители серии QCN, вместе охватывающие диапазон от 330 до 4500 МГц, обладают одинаковыми посадочными местами, поэтому разработчики могут менять характеристики дифференциальной схемы усилителя по своему желанию, всего лишь заменяя квадратурный гибридный ответвитель, СВч МИС усилителя, а также изменяя соответствующим образом схему смещения.

Нужно также отметить, что в рассмотренной в статье дифференциальной схеме применяется два отдельных усилителя GVA-91+. Характеристики схемы могут быть дополнительно улучшены за счет использования двух усилителей в одном корпусе. Компания Mini-Circuits начала разработку компонента с двумя кристаллами GVA-91+. Это позволит улучшить согласование усилителей за счет сведения к минимуму влияния небольших отклонений производственного процесса. 